\title{
Putative physiological mechanisms underlying tDCS analgesic effects
}

\author{
Helena Knotkova ${ }^{1,2 *}$, Michael A. Nitsche ${ }^{3}$ and Ricardo A. Cruciani ${ }^{1,2,4}$ \\ ${ }^{1}$ Department of Pain Medicine and Palliative Care, Institute for Non-Invasive Brain Stimulation, Research Division, Beth Israel Medical Center, New York, NY, USA \\ ${ }^{2}$ Department of Neurology, Albert Einstein College of Medicine, Bronx, NY, USA \\ ${ }^{3}$ Department of Clinical Neurophysiology, Georg-August-University, Goettingen, Germany \\ ${ }^{4}$ Department of Anesthesiology, Albert Einstein College of Medicine, Bronx, NY, USA
}

\section{Edited by:}

Marom Bikson, The City College of New York of The City University of New York, USA

Reviewed by:

Vince P. Clark, The Mind Research

Network, USA

Asif Rahman, The City College of The

City University of New York, USA

\section{*Correspondence:}

Helena Knotkova, Department of Pain Medicine and Palliative Care, Institute for Non-Invasive Brain Stimulation,

Beth Israel Medical Center, 120 E 16th

Street, 12th Floor, New York, NY

10003, USA

e-mail:HKnotkov@chpnet.org
Transcranial direct current stimulation (tDCS) is a non-invasive neuromodulation technique that induces changes in excitability, and activation of brain neurons and neuronal circuits. It has been observed that beyond regional effects under the electrodes, tDCS also alters activity of remote interconnected cortical and subcortical areas. This makes the tDCS stimulation technique potentially promising for modulation of pain syndromes. Indeed, utilizing specific montages, tDCS resulted in analgesic effects in experimental settings, as well as in post-operative acute pain and chronic pain syndromes. The promising evidence of tDCS-induced analgesic effects raises the challenging and complex question of potential physiologic mechanisms that underlie/mediate the accomplished pain relief. Here we present hypotheses on how the specific montages and targets for stimulation may affect the pain processing network.

Keywords: transcranial direct current stimulation (tDCS), pain, analgesia, mechanisms, neuromodulation
Transcranial direct current stimulation (tDCS) is a noninvasive neuromodulation technique (Nitsche and Paulus, 2000) that delivers electrical current of relatively low intensity ( 1 or 2 miliamperes over an area of about 20 to $35 \mathrm{~cm}^{2}$ ) painlessly through the skull to selected areas of the brain, and induce changes in excitability and activation of brain neurons and neuronal circuits. An important, and perhaps primary, mechanism of tDCS is a subthreshold modulation of neuronal resting membrane potential. Stimulation of severalminute duration results in a polarity-dependent induction of glutamatergic calcium-dependent neuroplasticity, which shares some aspects with long-term potentiation, and depression (Nitsche et al., 2003, 2008). The effects of tDCS on cortical excitability are polarity-dependent. Anodal tDCS enhances, while cathodal tDCS diminishes excitability, within certain parameters of stimulation duration and strength (Nitsche and Paulus, 2000, 2001; Nitsche et al., 2003). Too long, or strong stimulation, however, may have an opposite effect, resulting in diminished excitability after the anodal stimulation and enhanced excitability after the cathodal tDCS (Batsikadze et al., 2013; Monte-Silva et al., 2013). In addition, recent evidence suggests that tDCS interacts with various cerebral neurotransmitter systems, and is mediated by dopamine, acetylcholine, serotonin or GABA (Nitsche et al., 2004a,b,c, 2006, 2009; Kuo et al., 2007; Terney et al., 2008). Moreover, tDCS has been shown to facilitate changes in brain-derived neurotrophic factor (BDNF; Fritsch et al., 2010) that is a distinct marker of neuronal plasticity and notably has been associated with pain processing (Stefani et al., 2012).
The effects and outcome of tDCS depend on the area of the brain that is stimulated (e.g., Nitsche et al., 2007). Beyond regional effects under the electrodes, activity alterations of interconnected remote cortical and subcortical areas have also been described (Polania et al., 2011; DaSilva et al., 2012). This makes the tDCS stimulation technique potentially promising for modulation of pain syndromes, which include pathological alterations of activity, and excitability of a multitude of interconnected areas. Different interwoven cortico-subcortical pain-related networks, so-called Pain Matrix, cover sensory-discriminative, affective, and vegetative aspects of pain processing. The main components of the sensory-discriminative pain processing network are the spinothalamic tract, the lateral thalamus, somatosensory areas, and the posterior insula (Moisset and Bouhassira, 2007). The affective component of pain has been related to anterior insular, and cingulate cortices, as well as prefrontal areas. Vegetative, and neuroendocrine effects of pain perception are closely linked to various subcortical regions, such as amygdala, hypothalamus ventral tegmental area and others (Hsieh et al., 1995; Zaghi et al., 2009). Neuroplastic alterations of connectivity between these areas might contribute to chronification of pain.

Several specific tDCS montages have been probed, which resulted in analgesic effects: (a) excitability-enhancing (anodal) tDCS delivered over the primary motor cortex (e.g., Fregni et al., 2006a,b; Fenton et al., 2008; Kuhnl et al., 2008; Knotkova et al., 2013), typically with the anode positioned over M1 contralateral to the affected side and cathode over the ipsilateral supraorbital region in case of unilateral pain; or the anode over M1 of the 
dominant hemisphere and the cathode over the supraorbital region contralateral to the anode in case of bilateral pain; (b) excitability-diminishing (cathodal) tDCS over the somatosensory cortex (Antal et al., 2008; Knotkova et al., 2009) [the cathode over S1, the anode over the contralateral supraorbital region, with the same consideration of pain localization as described above]; (c) anodal tDCS over the left dorsolateral prefrontal cortex (DLPFC; Riberto et al., 2011; Valle et al., 2009) [the anode over DLPFC corresponding with the F3 electrode position of the 10-20 international EEG system, the cathode over the contralateral supraorbital region]; (d) combined anodal left DLPFC and cathodal tDCS of contralateral somatosensory cortex [the cathode over the gut representation area of the right S1] (Borckardt et al., 2011).

In the available studies, the assessment of analgesic effects elicited with these montages in subjects with bilateral pain has not systematically compared the pain intensity separately at each side, and thus it is unclear if the effect of the stimulation was unilateral or bilateral. However, as noted by Antal et al. (2010), there is evidence that tDCS of M1 induces widespread changes in cortical activity and can induce changes in activity of the contralateral hemisphere.

Analgesic effects have been explored in experimental settings (experimentally induced pain in healthy subjects), as well as in post-operative acute pain and chronic pain syndromes in clinical settings. The analgesic effects have been shown to be cumulative, and therefore a majority of clinical trials of tDCS encompassed the stimulation on several (usually 5, rarely 10 ) consecutive days. For example, anodal M1 tDCS over five consecutive days resulted in a significant decrease of pain intensity after spinal cord injury (Fregni et al., 2006a), and similar results were observed in chronic neuropathic pain due to multiple sclerosis (Mori et al., 2010), chronic pelvic pain (Fenton et al., 2009) and pain of various origin (Antal et al., 2010; Knotkova et al., 2013). Stimulation over the prefrontal cortex resulted in significant pain relief after 10 but not 5 sessions (Valle et al., 2009). Meta-analysis of analgesic effects in the existing studies is thoroughly discussed in a Cochrane systematic review by O'Connell et al. (2011). Overall, a significant heterogeneity among studies was noted, and a sub-group evaluation of tDCS applied to the motor cortex suggested superiority of active stimulation over sham (SMD $-0.59,95 \%$ CI -1.10 to -0.08 ).

The evidence of tDCS-induced analgesic effects raises the challenging and complex question of potential physiologic mechanisms that underlie/mediate the accomplished pain relief. Here we develop hypotheses on how the specific montages and targets for stimulation may affect the pain processing network.

\section{MODULATION OF THE SENSORY-DISCRIMINATIVE PAIN PROCESSING \\ CHANGES IN THALAMIC ACTIVITY}

Thalamic activity is crucial for processing and filtering of nociceptive signals on the pathways ascending to the cortical part of the pain matrix, and the thalamus also receives direct input from descending cortico-thalamic pathways originating in the primary motor cortex. Notably, anodal tDCS over M1 has been shown to increase functional coupling between ipsilateral M1 and thalamus (Polania et al., 2011) and therefore it is likely that the analgesic effects observed after the facilitatory motor cortex stimulation are at least partially attributable to modulation of thalamic activity. Indeed, changes in regional cerebral blood flow following epidural motor cortex stimulation (Peyron et al., 1995; Garcia-Larrea et al., 1999; Garcia-Larrea and Peyron, 2007) indicated that stimulation of the motor cortex may trigger rapid and phasic activation in the lateral thalamus (which receives direct input from the motor area), followed by parallel or secondary activation of medial thalamic regions, and the anterior cingulate gyrus, the insula and the upper brain stem. (Garcia-Larrea et al., 1999). Interestingly, the blood flow change in the lateral thalamus has not significantly correlated with patient's perceived pain relief and although important as a trigger of further events, the activation of the lateral thalamus is not a sufficient condition for clinical pain-relieving effects (Garcia-Larrea et al., 1999). However, neuronal inactivation in response to motor cortex stimulation was detected in thalamic sensory neurons, specifically in ventral posterolateral nuclei and centromedian-parafascicular thalamic complex, and the inactivating effect was particularly observed for neurons responsive to nociceptive peripheral stimulation (Pagano et al., 2012). It can be speculated that the inhibition of the sensory thalamic nuclei and the activation of the lateral (motoric) thalamic area after the motor cortex stimulation may be functionally related, the lateral thalamus receiving the input from the motor cortex and inhibiting the thalamic sensory neurons that are involved in the transmission of nociceptive signals from the periphery.

\section{MOTOR-CORTEX-DRIVEN INHIBITION OF THE SOMATOSENSORY CORTEX}

As there is a direct connection between the primary motor cortex and primary somatosensory cortex via cortico-cortical pathways in the human brain, it is possible that stimulation of the motor cortex directly inhibits the activity in the somatosensory cortex. By these means, recent work by Chiou et al. (2012) on animal models demonstrated that motor cortex stimulation blocked the transmission of somatosensory information to the primary somatosensory cortex. In the experiment, epidural motor cortex stimulation, but not stimulation outside of the motor cortex, lead to suppression of the ipsilateral somatosensory evoked potentials. However, these findings have to be interpreted with caution as the stimulation was delivered at suprathreshold level, and therefore the effects cannot be directly extrapolated to the subthreshold tDCS stimulation. Interestingly, a study of the somatosensory cortex in rats (Choi and Callaway, 2011) has shown the existence of inhibitory neurons in the somatosensory cortex that receive direct monosynaptic input not only from distant areas such as thalamus, but also from the ipsilateral motor cortex.

\section{DIRECT INHIBITION OF THE SOMATOSENSORY CORTEX}

Cathodal tDCS is thought to have a direct excitability-reducing effect on the S1 area. Since hyperexcitability within S1 in chronic pain syndromes, such as facial neuropathic pain or carpal tunnel syndrome has been clearly documented in recent neuroimaging studies, $\mathrm{tDCS}$-generated reduction of this pathological excitability alteration should be beneficial. Moreover, thickening of neuronal 
layers in the somatosensory cortex has been observed in chronic migraineurs (DaSilva et al., 2007), which might be a hint for structural neuroplastic alterations of the respective area due to its pain-related hyperactivity of this area. It has been suggested that repeated/long-term down-regulation of nociceptive activity in S1, which could be also induced by tDCS, may result in normalization of this maladaptive change.

\section{MODULATION OF THE EMOTIONAL/AFFECTIVE COMPONENT OF PAIN \\ ACTIVATION OF THE PREFRONTAL CORTEX}

Stimulation of the prefrontal cortex has been associated with a modulation of a large neuronal network related to the limbic system, including the cingulate gyrus and parahippocampal areas (Mottaghy et al., 2000; Catafu et al., 2001). The dopaminergic and serotoninergic circuits of the frontal and prefrontal cortex and related subcortical areas mediate attentional control, impulsivity, working memory, decision-making, as well as mood regulation and emotional processing. Notably, activation of the brain structures associated with emotional appraisal of pain in condition of the epidural motor cortex stimulation correlated with subjectively reported pain relief (Peyron et al., 1995; Garcia-Larrea et al., 1999) and it is thought that neuromodulation modifying emotional appraisal of pain and pain experience is directly related to clinical analgesic effects of the neuromodulatory interventions (GarciaLarrea et al., 1999). Indeed, tDCS stimulation of the prefrontal dorsolateral cortex increased pain thresholds in healthy subjects (Boggio et al., 2008) and relieved chronic pain (Valle et al., 2009).

\section{OPIOID RELEASE}

Interestingly, a recent work by DosSantos et al. (2012) has shown that a single session of anodal tDCS over the motor cortex results in reduction of mu opioid receptor binding of an exogenous receptor ligand in the pain matrix, suggesting that the analgesic effect of M1-tDCS may possibly be due to a direct increase of endogenous opioid release (DosSantos et al., 2012). The authors suggest that the decreased binding of the exogenous ligand was possibly due to receptor occupancy by enhanced release of endogenous opioids. The reduction was detected in numerous cortical and subcortical structures of the pain matrix, such as nucleus accumbens, anterior cingulate cortex, insula and thalamus, and was accompanied by an increased threshold for experimentally induced cold pain. Although opioid analgesic effects are known to relate to both the emotional- as well as

\section{REFERENCES}

Antal, A., Brepohl, N., Poreisz, C., Boros, K., Csifcsak, G., and Paulus, W. (2008). Transcranial direct current stimulation over somatosensory cortex decreases experimentally induced acute pain perception. Clin. J. Pain 24, 56-63. doi: 10.1097/ajp. 0b013e318157233b

Antal, A., Terney, D., Kuhnl, S., and Paulus, W. (2010). Anodal transcranial direct current stimulation of the

sensory dimension of pain, no significant changes in clinical pain levels were elicited after a single tDCS session, suggesting that the immediate opiodergic effects of a single tDCS application are subclinical, and repeated application might be necessary to get clinically meaningful results.

\section{CONCLUSIONS AND IMPLICATIONS FOR FUTURE RESEARCH}

The findings suggest that multiple physiologic mechanisms mediate the analgesic effects of tDCS, involving changes in both the perceptual processing of pain and the emotional component of pain experience. However, the mechanisms and their translation into predicable clinical outcomes are far from being fully understood. Future studies are needed to expand understanding of tDCS-induced analgesic mechanisms and to address the presented hypotheses of tDCS effects on the pain-processing network. Extrapolating from studies of the epidural motor cortex stimulation, changes of the thalamic activation after tDCS may be determined via the regional cerebral blood flow evaluation in the thalamus and related regions after a single- and multiple tDCS stimulation of the motor cortex, including explorations of the association between the thalamic activation changes and pain relief. Future studies addressing the hypothesis of the tDCS-generated analgesic effects due to motor-cortex-driven inhibition of the somatosensory cortex may utilize evaluations of the somatosensory potentials, exploring suppression of the somatosensory evoked potentials after the anodal tDCS stimulation of the ipsilateral motor cortex. Moreover, studies of tDCS combined with functional imaging (fMRI) with regard to the inhibitory (cathodal) stimulation of the somatosensory cortex as well as the anodal tDCS in both the experimentally induced- and spontaneous chronic pain may provide further insight into the tDCS effects on the pain matrix. Beyond the exploration of regional effects, functional imaging data might also be helpful to explore stimulation-dependent alterations of the pain-related cerebral network, via functional connectivity analysis. The latter approach will be also relevant to explore specific effects of different stimulation paradigms on the above-mentioned discernable components of the pain matrix.

Further, future studies are needed to systematically elucidate the impact of the stimulation parameters on the analgesic outcomes, including aspects related to stimulation intensity, strength, repetition rate and timing, as well as electrode positions and stimulation polarity, because a critical aspect of the future impact of tDCS in pain management is the optimization of the stimulation protocols with regard to specific patient populations.

2000. doi: 10.1113/jphysiol.2012. 249730

Boggio, P. S., Zaghi, S., Lopes, M., and Fregni, F. (2008). Modulatory effects of anodal transcranial direct current stimulation on perception and pain thresholds in healthy volunteers. Eur. J. Neurol. 15, 1124-1130. doi: 10.1111/j.14681331.2008.02270.x

Borckardt, J. J., Romagnuolo, J., Reeves, S. T., Madan, A., Frohman, H.,
Beam, W., et al. (2011). Feasibility, safety, and effectiveness of transcranial direct current stimulation for decreasing post-ERCP pain: a randomized, sham-controlled, pilot study. Gastrointest. Endosc. 73, 1158-1164. doi: 10.1016/j.gie.2011. 01.050

Catafu, A. M., Perez, V., Gironell, A., Martin, J. C., Kulisevsky, J., Estorch, M., et al. (2001). SPECT mapping of cerebral activity changes induced 
by repetitive transcranial magnetic stimulation in depressed patients. Psychiatry Res. 106, 151160. doi: $10.1016 / 50925-4927(01)$ 00079-8

Chiou, R. J., Lee, H. Y., Chang, C. W., Lin, K. H., and Kuo, C. C. (2012). Epidural motor cortex stimulation suppresses somatosensory evoked potentials in the primary somatosensory cortex of the rat. Brain Res. 1463, 42-50. doi: 10. 1016/j.brainres.2012.04.027

Choi, J., and Callaway, E. M. (2011). Monosynaptic inputs to ErbB4expressing inhibitory neurons in mouse primary somatosensory cortex. J. Comp. Neurol. 519, 34023414. doi: $10.1002 / \mathrm{cne} .22680$

DaSilva, A. F., Granziera, C., Snyder, J., and Hadjikhani, N. (2007). Thickening in the somatosensory cortex of patients with migraine. Neurology 69, 1990-1995. doi: $10.1212 / 01 . w n l .0000291618$. 32247.2d

DaSilva, A. F., Mendonca, M. E., Zaghi, S., Lopes, M., DosSantos, M. F., Spierings, E. L., et al. (2012). tDCS-induced analgesia and electrical fields in painrelated neural networks in chronic migraine. Headache 52, 1283-1295. doi: $\quad 10.1111 / j .1526-4610.2012$. 02141.x

DosSantos, M. F., Love, T. M., Martikainen, I. K., Nascimento, T. D., Fregni, F., Cummiford, C., et al. (2012). Immediate effects of tDCS on the mu-opioid system of a chronic patient. Front. Psychiatry 3:93. doi: 10.3389/fpsyt.2012. 00093

Fenton, B., Fanning, J., Boggio, P., and Fregni, F. (2008). A pilot efficacy trial of tDCS for the treatment of refractory chronic pelvic pain. Brain Stimul. 1, 260. doi: 10.1016/j.brs. 2008.06.044

Fenton, B. W., Palmieri, P. A., Boggio, P., Fanning, J., and Fregni, F. (2009). A preliminary study of transcranial direct current stimulation for the treatment of refractory chronic pelvic pain. Brain Stimul. 2, 103 107. doi: 10.1016/j.brs.2008.09.009

Fregni, F., Boggio, P. S., Lima, M. C., Ferreira, M. J., Wagner, T., and Rigonatti, S. P. (2006a). A shamcontrolled, phase II trial of transcranial direct current stimulation for the treatment of central pain in traumatic spinal cord injury. Pain 122, 197-209. doi: 10.1016/j.pain. 2006.02.023

Fregni, F., Gimenes, R., Valle, A. S., Ferreira, M. J., Rocha, R. R., Natalle, L., et al. (2006b). A randomized, sham- controlled, proof of principle study of transcranial direct current stimulation for the treatment of pain in fibromyalgia. Arthritis Rheum. 54, 3988-3998.

Fritsch, B., Reis, J., Martinowich, K., Schambra, H. M., Ji, Y., and Cohen, L. G. (2010). Direct current stimulation promotes BDNF-dependent synaptic plasticity: potential implications for motor learning. Neuron 66, 198-204. doi: 10.1016/j.neuron. 2010.03.035

Garcia-Larrea, L., and Peyron, R. (2007). Motor cortex stimulation for neuropathic pain: from phenomenology to mechanisms. Neuroimage 37, S71-S79. doi: 10. 1016/j.neuroimage.2007.05.062

Garcia-Larrea, L., Peyron, R., Mentens, P., Gregoire, M. C., Lavenne, F., Le Bars, D., et al. (1999). Electrical stimulation of motor cortex for pain control: a combined PETscan and electrophysiological study. Pain 259-273. doi: 10.1016/s03043959(99)00114-1

Hsieh, J. C., Belfrage, M., StoneElander, M., Hansson, P., and Ingvar, M. (1995). Central representation of chronic ongoing neuropathic pain studies by positron emission tomography. Pain 63, 225-236. doi: 10.1016/0304-3959(95)00048-w

Knotkova, H., Homel, P., and Cruciani, R. A. (2009). Cathodal tDCS over the somatosensory cortex relieved chronic neuropathic pain in a patient with complex regional pain syndrome (CRPS/RSD). Case Report. J. Pain Manag. 2, $365-$ 368. doi: 10.1016/j.jpain.2009. 01.164

Knotkova, H., Leuschner, Z., Soto, E., Greenberg, A., and Cruciani, R. A. (2013). Evaluation of outcomes from Transcranial Direct Current Stimulation (tDCS) for the treatment of chronic pain. J. Pain 14, S64.

Kuhnl, S., Terney, D., Paulus, W., and Antal, A. (2008). The effect of daily sessions of anodal tDCS on chronic pain. Brain Stimul. 1, 281. doi: 10. 1016/j.brs.2008.06.249

Kuo, M. F., Grosch, J., Fregni, F., Paulus, W., and Nitsche, M. A. (2007). Focusing effect of acetylcholine on neuroplasticity in the human motor cortex. J. Neurosci. 27, 14442-14447. doi: 10. 1523/jneurosci.4104-07.2007

Monte-Silva, K., Kuo, M. F., Hessenthaler, S., Fresnoza, S., Liebe$\tan z$, D., Paulus, W., et al. (2013). Induction of late LTP-like plasticity in the human motor cortex by repeated non-invasive brain stimu- lation. Brain Stimul. 6, 424-432. doi: 10.1016/j.brs.2012.04.011

Moisset, X., and Bouhassira, D. (2007). Brain imaging of neuropathic pain. Neuroimage 37(Suppl. 1), S80-S88. doi: 10.1016/j.neuroimage.2007.03. 054

Mori, F., Codeca, C., Kusayanagi, H., Monteleone, F., Buttari, F., Fiore, S., et al. (2010). Effects of anodal transcranial direct current stimulation on chronic neuropathic pain in patients with multiple sclerosis. J. Pain 11, 436-442. doi: 10.1016/j. jpain.2009.08.011

Mottaghy, F. M., Krause, B. J., Kemna, L. J., Topper, R., Tellmann, L., and Beu, M. (2000). Modulation of the neuronal circuitry subserving working memory in healthy human subjects by repetitive transcranial magnetic stimulation. Neurosci. Lett. 280, 167-170. doi: 10.1016/s03043940(00)00798-9

Nitsche, M. A., Cohen, L. G., Wassermann, E. M., Priori, A., Lang, N., Antal, A., et al. (2008). Transcranial direct current stimulation: state of the art 2008. Brain Stimul. 1, 206-223. doi: 10.1016/j.brs.2008. 06.004

Nitsche, M. A., Doemkes, S., Karakose, T., Antal, A., Liebetanz, D., Lang, N., et al. (2007). Shaping the effects of transcranial direct current stimulation of the human motor cortex. J. Neurophysiol. 97, 3109-3117. doi: 10.1152/jn.01312. 2006

Nitsche, M. A., Fricke, K., Henschke, U., Schlitterlau, A., Liebetanz, D., Lang, N., et al. (2003). Pharmacological modulation of cortical excitability shifts induced by transcranial direct current stimulation in humans. J. Physiol. 553, 293-301. doi: 10.1113/jphysiol.2003.049916

Nitsche, M. A., Grundey, J., Liebe$\operatorname{tanz}$, D., Lang, N., Tergau, F., and Paulus, W. (2004a). Catecholaminergic consolidation of motor cortical neuroplasticity in humans. Cereb. Cortex 14, 1240-1245. doi: 10. 1093/cercor/bhh085

Nitsche, M. A., Jaussi, W., Liebetanz, D., Lang, N., Tergau, F., and Paulus, W. (2004b). Consolidation of human motor cortical neuroplasticity by Dcycloserine. Neuropsychopharmacology 29, 1573-1578. doi: 10.1038/sj. npp. 1300517

Nitsche, M. A., Kuo, M. F., Karrasch, R., Wächter, B., Liebetanz, D., and Paulus, W. (2009). Serotonin affects transcranial direct current-induced neuroplasticity in humans. Biol. Psychiatry $66,503-508$. doi: $10.1055 / \mathrm{s}$ 0029-1238466
Nitsche, M. A., Lampe, C., Antal, A., Liebetanz, D., Lang, N., Tergau, F., et al. (2006). Dopaminergic modulation of long-lasting direct currentinduced cortical excitability changes in the human motor cortex. Eur. J. Neurosci. 23, 1651-1657. doi: 10. 1111/j.1460-9568.2006.04676.x

Nitsche, M. A., Liebetanz, D., Antal, A., Lang, N., Tergau, F., and Paulus, W. (2003). Modulation of cortical excitability by weak direct current stimulation-technical, safety and functional aspects. Suppl. Clin. Neurophysiol. 56, 255-276. doi: 10.1016/S1567424X(09)70230-2

Nitsche, M. A., Liebetanz, D., Schlitterlau, A., Henschke, U., Fricke, K., Frommann, K., et al. (2004c). GABAergic modulation of DC stimulation-induced motor cortex excitability shifts in humans. Eur. J. Neurosci. 19, 2720-2726. doi: $\quad 10.1111 / j .0953-816 X .2004$. 03398.x

Nitsche, M. A., and Paulus, W. (2000). Excitability changes induced in the human motor cortex by weak transcranial direct current stimulation. J. Physiol. 527, 633-639. doi: $\quad 10.1111 /$ j.1469-7793.2000 t01-1-00633.x

Nitsche, M. A., and Paulus, W. (2001). Sustained excitability elevations induced by transcranial DC motor cortex stimulation in humans. Neurology 57, 1899-1901. doi: 10. 1212/wnl.57.10.1899

O'Connell, N. E., Wand, B. M., Marston, L., Spencer, S., and Desouza, L. H. (2011). Non-invasive brain stimulation techniques for chronic pain. A report of a Cochrane systematic review and meta-analysis. Eur. J. Phys. Rehabil. Med. 47, 309-326.

Pagano, R. L., Fonoff, E. T., Dale, C. S., Ballester, G., Teixeira, M. J., and Britto, L. R. (2012). Motor cortex stimulation inhibits thalamic sensory neurons and enhances activity of PAG neurons: possible pathways for antinociception. Pain 153, 23592369. doi: 10.1016/j.pain.2012. 08.002

Peyron, R., Garcia-Larrea, L., Deiber, M. P., Cinotti, L., Convers, P., Sindou, M., et al. (1995). Electrical stimulation of precentral cortical area in the treatment of central pain: electrophysiological and PET study. Pain 62, 275286. doi: 10.1016/0304-3959(94) 00211-v

Polania, R., Paulus, W., and Nitsche, M. A. (2011). Modulating corticostriatal and thalamo-cortical func- 
tional connectivity with transcranial direct current stimulation. Hum. Brain Mapp. 33, 2499-2508. doi: 10. 1002/hbm. 21380

Riberto, M., Marcon Alfieri, F., Monteiro de Benedetto Pacheco, K., Dini Leite, V., Nemoto Kaihami, H., Fregni, F., et al. (2011). Efficacy of transcranial direct current stimulation coupled with a multidisciplinary rehabilitation program for the treatment of fibromyalgia. Open Rheumatol. J. 5, 4550. doi: 10.2174/18743129011050 10045

Stefani, L. C., Torres, I. L., de Souza, I. C., Rozisky, J. R., Fregni, F., and Caumo, W. (2012). BDNF as an effect modifier for gender effects on pain thresholds in healthy subjects.
Neurosci. Lett. 514, 62-66. doi: 10 . 1016/j.neulet.2012.02.057

Terney, D., Bergmann, I., Poreisz, C., Chaieb, L., Boros, K., Nitsche, M. A., et al. (2008). Pergolide increases the efficacy of cathodal direct current stimulation to reduce the amplitude of laser-evoked potentials in humans. J. Pain Symptom Manage. 36, 79-91. doi: 10.1016/j. jpainsymman.2007.08.014

Valle, A., Roizenblatt, S., Botte, S., Zaghi, S., Riberto, M., Tufik, S., et al. (2009). Efficacy of anodal transcranial direct current stimulation (tDCS) for the treatment of fibromyalgia: results of a randomized, sham-controlled longitudinal clinical trial. J. Pain Manag. 2, 353361 .
Zaghi, S., Heine, N., and Fregni, F. (2009). Brain stimulation for the treatment of pain: a review of costs, clinical effects, and mechanisms of treatment for three different central neuromodulatory approaches. J. Pain Manag. 2, 339-352.

Conflict of Interest Statement: The authors declare that the research was conducted in the absence of any commercial or financial relationships that could be construed as a potential conflict of interest.

Received: 17 June 2013; paper pending published: 18 July 2013; accepted: 10 September 2013; published online: 26 September 2013.
Citation: Knotkova H, Nitsche MA and Cruciani RA (2013) Putative physiological mechanisms underlying tDCS analgesic effects. Front. Hum. Neurosci. 7:628. doi: 10.3389/fnhum.2013.00628 This article was submitted to the journal Frontiers in Human Neuroscience. Copyright (c) 2013 Knotkova, Nitsche and Cruciani. This is an open-access article distributed under the terms of the Creative Commons Attribution License (CC BY). The use, distribution or reproduction in other forums is permitted, provided the original author(s) or licensor are credited and that the original publication in this journal is cited, in accordance with accepted academic practice. No use, distribution or reproduction is permitted which does not comply with these terms. 